\title{
Exceptional Points through Variation of Distances between Four Coaxial Dielectric Disks
}

\author{
Konstantin Pichugin ${ }^{1}\left[\right.$, Almas Sadreev ${ }^{1,2, * \mathbb{C}}$ and Evgeny Bulgakov ${ }^{1}$ \\ 1 Federal Research Center KSC SB RAS, Kirensky Institute of Physics, 660036 Krasnoyarsk, Russia; \\ knp@tnp.krasn.ru (K.P.); bulgakov100@yandex.ru (E.B.) \\ 2 Department of Electronic Engineering, College of Information Science and Technology, Jinan University, \\ Guangzhou 510632, China \\ * Correspondence: almas@tnp.krasn.ru
}

\section{check for}

updates

Citation: Pichugin, K.; Sadreev, A. Bulgakov, E. Exceptional Points through Variation of Distances between Four Coaxial Dielectric Disks. Photonics 2021, 8, 460. https://doi.org/10.3390/ photonics 8110460

Received: 4 August 2021

Accepted: 15 October 2021

Published: 21 October 2021

Publisher's Note: MDPI stays neutral with regard to jurisdictional claims in published maps and institutional affiliations.

Copyright: (c) 2021 by the authors. Licensee MDPI, Basel, Switzerland. This article is an open access article distributed under the terms and conditions of the Creative Commons Attribution (CC BY) license (https:// creativecommons.org/licenses/by/ $4.0 /)$.

\begin{abstract}
By variation of a refractive index and aspect ratio of the isolated disk, we achieved exceptional points (EPs) at which the resonant frequencies and resonant modes coalesce. However, in practice, that kind of variation presents a technological problem. We considered the method to avoid the problem by substitution of two disk's dimers. In each dimer, variation of the distance between disks was equivalent to a variation of the aspect ratio of the dimer. Moreover, the variation of the distance between dimers provides the second parameter that gives rise to a vast number of EPs. We recovered the initial resonant eigenmode by encircling multiple EPs two, three, and four times in the two-dimensional parametric space of distances.
\end{abstract}

Keywords: resonant eigenmodes; multiple exceptional points; encircling of exceptional points

\section{Introduction}

A dielectric particle embedded into open space is specified by eigenfrequencies, which are complex owing to open boundary conditions for the solutions of the homogeneous Maxwell equations. The most drastic difference between closed systems and open systems is that the latter exhibit exceptional points (EPs) where both the complex eigenfrequencies and eigenmodes coalesce [1,2]. Many works on EPs and their applications are associated with parity-time (PT) symmetric optical systems with a balanced gain and loss. In that case, EPs can be easily found by tuning a single parameter, namely, the amplitude of the balanced gain and loss [3-8]. Since it is not always easy or desirable to keep a balanced gain and loss, it is of significant interest to explore EPs and their applications in non-PT-symmetric optical systems. In the photonic system, the appearance of EPs can be exploited to a broad range of interesting applications, including lasing [9], asymmetric mode switching [10], nonreciprocal light transmission [11,12], and ultrasensitive sensing [13].

Currently, there have been studies concerning EPs for resonant states in extended periodic dielectric structures sandwiched between two homogeneous half-spaces [14-17], dual-mode planar optical waveguides [10] and plasmonic waveguides [18], layered structures [3,19], two infinitely long dielectric cylinders [20-24], and even a single rod with a deformed cross-section [22,25-28]. As for compact dielectric resonators, we distinguished the studies of EPs in the high-Q microcavities with their boundary shape continuous variables: a 2d chaotic deformed billiard [29], a compact-coated dielectric sphere [30], and a spheroid [31]. However, in practice, an achievement of the EP in such compact optical cavity by continuous deformation of its shape is technologically challenging.

In the present study, we considered two dimers that each consisted of two coaxial disks. This introduced two-fold scale parameters: the distance between the disks in each dimer and the distance between the dimers. The first scale introduced a coupling strength between disks in a dimer, and the second scale introduced a coupling strength between distant dimers. This approach made it easy to conduct experimentally two-fold variation 
in order to achieve EP as it was exploited first for experimental evidence of EPs in a system of three and more paired gold nanobars moving relative to each other [32]. Moreover, owing to hierarchical couplings between disks, we found a vast number of EPs encircling, of which there were open topological properties of multiple EPs [20,33,34]. In the photonic system, the appearance of EPs can be exploited to a broad range of interesting applications, including lasing [9], asymmetric mode switching [10], nonreciprocal light transmission [11,12], enhancement of the spontaneous emission [35], and ultrasensitive sensing [13].

\section{Exceptional Points in Single Disk}

Because of the rotational symmetry of the disk, the azimuthal index $m$ is preserved. That allows calculating the resonant frequencies and resonant eigenmodes separately in each sector of the azimuthal index $m$. Moreover, in the sector $m=0$, the TE and TM solutions of the homogeneous Maxwell equations are separated. In what follows, we restricted ourselves by the sector $m=0$, TE polarization. The separated disk was specified by a discrete series of the complex eigenfrequencies $k_{n}$ and the eigenmodes [36,37], which were calculated by COMSOL Multiphysics. The mesh was chosen as 20 nodes per wavelength, which is sufficient for numerics. The thickness of PML was chosen around the wavelength.

Previously, disk-shaped optical resonators have attracted much of interest primarily because of the revolutionary enhancement of the $Q$-factor by the engineering of super cavity modes [36] with the following experimental observation [38,39]. The underlying principle is based on avoided crossing of two resonances of the dielectric disk for variation of its aspect ratio as a ratio of thickness to the radius of the disk. We show, in this section, that the dielectric disk has also potential to achieve EPs by variation in the refractive index and the aspect ratio of the disk. In Figure 1, we present numerical results that show the evolution of complex TE resonant frequencies for variation in the aspect ratio at a fixed dielectric permittivity $\epsilon=12$ (a silicon disk) in the sector $m=0$. The EP was achieved at $h / a=0.725, \epsilon=9.8$ and $\operatorname{Re}(k a) \approx 2.7$ where the eigenmodes coalesce, as the insets in Figure 1 demonstrate.

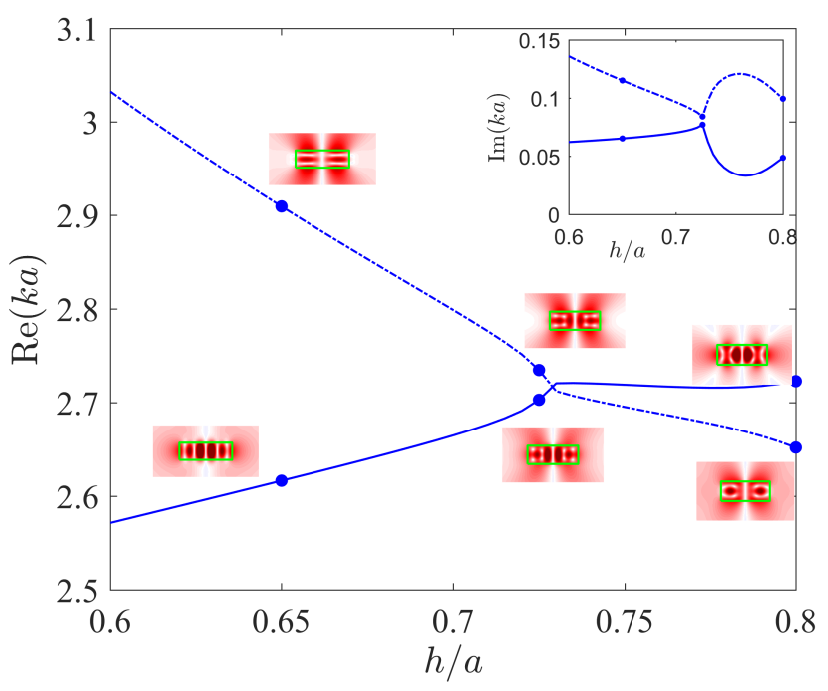

Figure 1. Evolution of complex TE resonant frequencies in single dielectric disk with the permittivity $\epsilon=12$ in the sector $m=0$ for variation of aspect ratio $h / a$ where $a$ is the radius and $h$ is the thickness of the disk. The insets show the azimuthal component $\left|E_{\phi}\right|$ of the electric field.

\section{Two-Scale Variation of Distances in the System of Four Coaxial Disks}

However, a tuning of a single dielectric disk-shaped resonator over the refractive index and aspect ratio presents a challenge for experimenters. We advocate a different method to achieve EPs by variation of two distances in a system of four coaxial silicon disks with a fixed refractive index $\epsilon=12$ arranged in two identical dimers as sketched in Figure 2. Each 
dimer consists of two coaxial disks with a fixed aspect ratio $h / a=1.04$ where $h$ and $a$ are the thickness and radius of the disk, respectively. Then, a change in the distance between disks in the dimer $D$ effectively can be considered as a variation of an aspect ratio of the dimer. The distance $L$ between the dimers gives the second parameter. Distances $L$ and $D$ provide a two-dimensional parametric space. In what follows, all scales are used to be dimensionless through the radius $a$. As seen from Figure 2, in the array of four coaxial disks, we had, in general, four distances $X=L+h, D+h, L+D+2 h$, and $L+2 D+3 h$, which defined four complex couplings between the disks. If the $n$-th elementary resonance of the isolated disk was well separated from the others, the truncated effective non-Hermitian Hamiltonian is defined by the couplings:

$$
V(X) \sim \frac{e^{-i k X}}{X^{2}}
$$

provided that $X \gg 1[37,40,41]$ and can be approximated as follows:

$$
H=\left(\begin{array}{cccc}
k_{n} a & V(D+h) & V(D+L+2 h) & V(2 D+L+3 h) \\
V(D+h) & k_{n} a & V(L+h) & V(D+L+2 h) \\
V(D+L+2 h) & V(L+h) & k_{n} a & V(D+h) \\
V(2 D+L+3 h) & V(D+L+2 h) & V(D+h) & k_{n} a
\end{array}\right)
$$

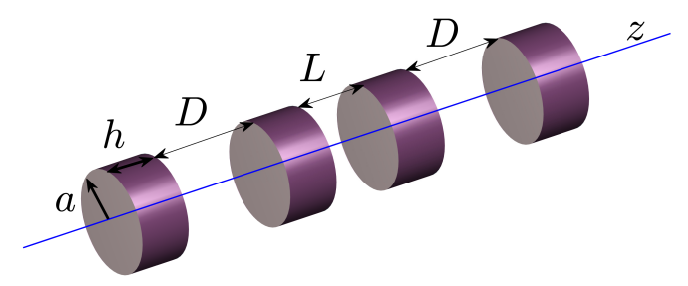

Figure 2. Four coaxial dielectric disks with $\epsilon=12$ and $h / a=1.04$ arranged in two dimers. The space parameters of disks and distances between them are given in terms of the disk radius $a$.

The eigenvalues of the Hamiltonian (2) define the eigenfrequencies of the system of four disks.

The eigenmodes of the isolated disk can be classified by the location of nodal surfaces. For the aspect ratio of the disk far from the avoided crossing of resonances, it can be described as the Fabry-Perot-type modes with nodal surfaces perpendicular to the $z$-axis and Mie-type modes with cylindrical nodal surfaces around the z-axis. Examples of these elementary resonant modes labeled as $1-5$ are shown in the insets of Figure 3 . In what follows, we study the parametric space $0.01 \leq L \leq 4$ and $0.01 \leq D \leq 4$. The examples of the evolution of the complex eigenfrequencies are presented in Figure 3 centered around resonances of the isolated disk shown by closed red circles and corresponding resonant modes in the insets. We see amazingly complicated and beautiful pictures, which in fact are the result of simple spiral behavior of four descending complex numbers $V(X)$ for increasing the distances $D$ and $L$. 

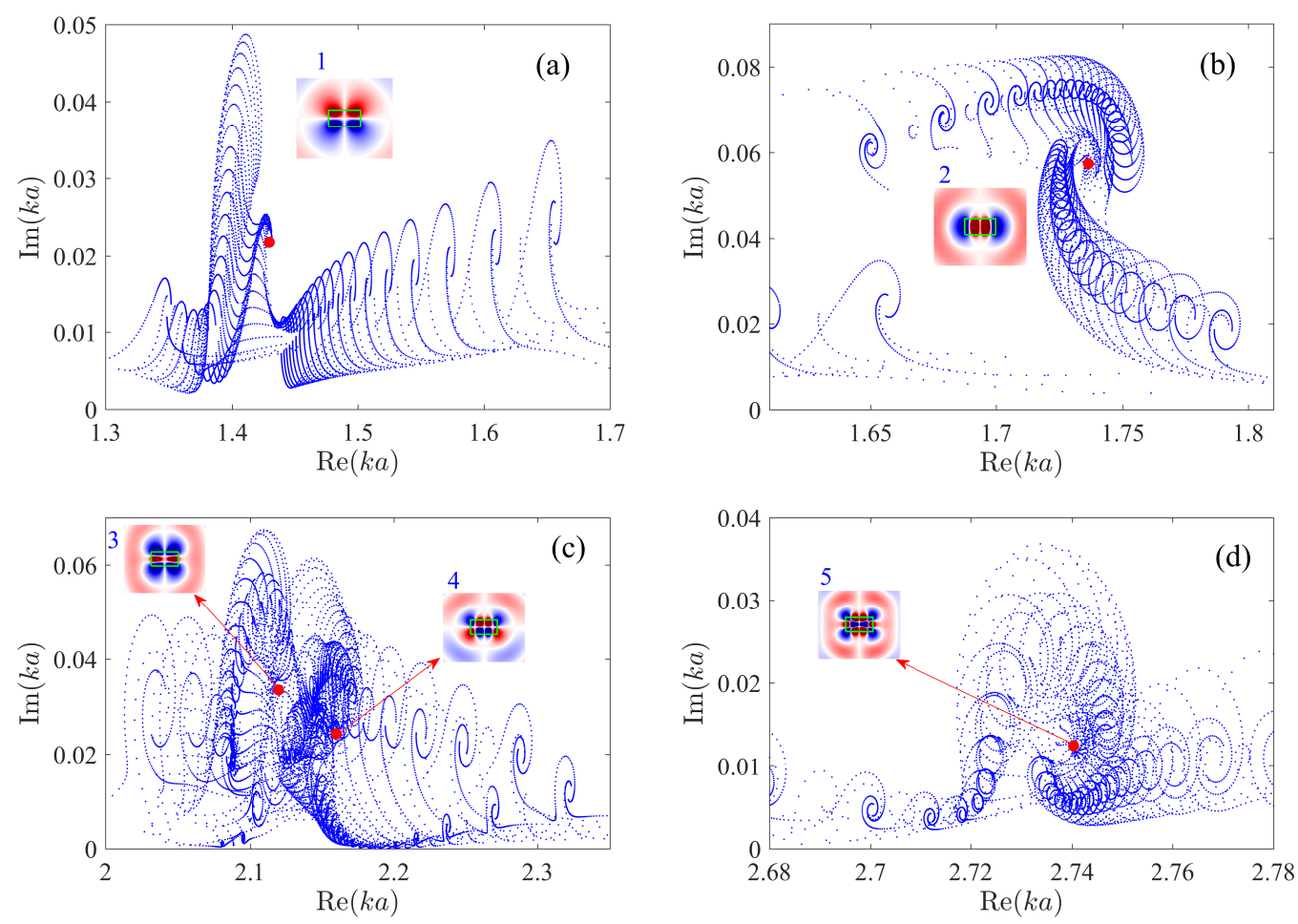

Figure 3. (a-d). Evolution of resonant frequencies of four disks for variation of two distances: the distance between dimers $0.05 \leq L \leq 4$ and the distance between disks in each dimer $0.05 \leq D \leq 4$. Red closed circles mark the eigenfrequencies of single disk.

\section{Exceptional Points in System of Four Disks}

It is clear that such a rich variety of evolution of resonant frequencies with two distances in the system of four disks proposes numerous events of EPs. Indeed, in the family of the eigenfrequencies that originated from the elementary mode 2 with the frequency $k_{1} a=1.75+0.057 i$, we revealed three EPs in the above given range of the distances, while the next family that originated from two close elementary modes 3 and 4 with the eigenfrequencies $k_{3} a=2.18+0.037 i$ and $k_{4} a=2.19+0.024 i$ resulted in a strikingly large number of 33 EPs. These EPs were classified by the symmetry of eigenmodes relative to $z \rightarrow-z$ with the eigenfrequencies and corresponding distance parameters listed in Table 1. Expansion of the ranges for distances $D$ and $L$ increased the number of EPs. Among the EPs listed in Table 1, we noted at the bottom of the table two anti-symmetric EPs whose resonances had extremely small resonant widths around $10^{-3}$. Figure 4 demonstrates that the EPs are the saddle points for evolution of the complex eigenfrequencies with variation of distances $L$ and $D$ shown by bold black solid lines. These saddles are unambiguous evidence of the existence of the EPs in the numerical data.

Often, EPs are points of measure zero in the eigenvalues of non-Hermitian Hamiltonian, which makes them very difficult to access, even with careful engineering. Nevertheless, their effect can be still felt globally. Particularly, an intriguing aspect of non-Hermitian systems is the eigenmode exchange along loops that trace closed trajectories around EPs. Figure 5 considers the evolution of resonant modes for encircling of symmetric EP and antisymmetric EP marked by closed and open circles by a rectangular close contour in the two-dimensional parametric space of distances $D, L$. These distances define an effective thickness of the dimer and the distance between dimers as shown in Figure 2. This encircling maps into closed trajectories in the complex plane of the eigenfrequencies $k a$ and the corresponding eigenmodes. Let us consider the symmetric EP marked by a closed circle. We start with the point $D=1, L=0.06$ marked by an asterisk in the inset of Figure 5a. In the first upward path shown by the blue arrow, we increased the distance between dimers from $L=0.06$ till $L=1.06$ and reached the point marked by a square. This upward 
path maps into the blue solid curve in the complex plane $k a$ originating from the point marked by an asterisk and terminating by the point marked by a square. We attached the corresponding symmetric eigenfunctions to these points. In the next horizontal path in the parametric space, we kept the distance between dimers constant but increased the distance between disks in dimers from $D=1$ till $D=2.5$ marked by a triangle. This horizontal path shown by the red arrow in the inset of Figure 5a maps into a red solid curve in the $k a$ complex plane. In the third downward path shown by the green arrow in the inset, we decreased the distance between dimers from $L=1.06$ till $L=0.06$ at $D=2.5$ marked by a rhombus. This path maps into a green curve in the plane $k a$. At last, we went back by a horizontal path shown by the gray arrow in the inset of Figure $5 \mathrm{a}$ to the initial point $D=1, L=0.06$. The path maps in $k a$ plane into gray curve originating by the rhombus and terminated by an asterisk. Although the paths in the parametric space $D, L$ were closed, the trajectory in the complex $k a$ plane came to a different point with a different eigenmode as seen from the Figure 5a, that is, the fundamental property of the encircling of the EP. In order to restore tie eigenfrequency and eigenfunction, we had to repeat once more the encircling as was first demonstrated by Dembowskii et al in a microwave metallic resonator [42]. EPs have also been observed experimentally in acoustic systems [34] and electrical circuits [43]. Figure $5 b$ shows the same phenomenon for the odd EP marked by an open circle in the inset of Figure.

Table 1. The eigenfrequencies of EPs with corresponding distances.

\begin{tabular}{cccccccc}
\hline \multicolumn{4}{c}{ Symmetric } & \multicolumn{4}{c}{ Anti-Symmetric } \\
\hline & $\boldsymbol{k} \boldsymbol{a}$ & $\boldsymbol{D}$ & $\boldsymbol{L}$ & & $\boldsymbol{k} \boldsymbol{c}$ & $\boldsymbol{D}$ & $\boldsymbol{L}$ \\
\hline 1 & $1.736+0.066 \mathrm{i}$ & 2.04 & 0.97 & 1 & $1.739+0.046 \mathrm{i}$ & 1.75 & 0.56 \\
2 & $2.030+0.006 \mathrm{i}$ & 0.14 & 0.07 & 2 & $1.734+0.060 \mathrm{i}$ & 3.20 & 2.35 \\
3 & $2.050+0.006 \mathrm{i}$ & 0.22 & 0.12 & 3 & $2.110+0.013 \mathrm{i}$ & 0.23 & 0.65 \\
4 & $2.152+0.044 \mathrm{i}$ & 0.55 & 1.67 & 4 & $2.145+0.020 \mathrm{i}$ & 0.46 & 0.07 \\
5 & $2.145+0.018 \mathrm{i}$ & 1.06 & 0.26 & 5 & $2.152+0.036 \mathrm{i}$ & 0.67 & 3.38 \\
6 & $2.139+0.043 \mathrm{i}$ & 1.25 & 1.18 & 6 & $2.147+0.019 \mathrm{i}$ & 0.72 & 1.63 \\
7 & $2.150+0.026 \mathrm{i}$ & 1.26 & 2.79 & 7 & $2.141+0.035 \mathrm{i}$ & 1.00 & 2.65 \\
8 & $2.095+0.032 \mathrm{i}$ & 1.29 & 0.50 & 8 & $2.175+0.028 \mathrm{i}$ & 1.53 & 0.35 \\
9 & $2.106+0.013 \mathrm{i}$ & 1.56 & 0.23 & 9 & $2.133+0.028 \mathrm{i}$ & 2.20 & 0.90 \\
10 & $2.139+0.023 \mathrm{i}$ & 1.69 & 1.43 & 10 & $2.133+0.025 \mathrm{i}$ & 2.32 & 1.96 \\
11 & $2.096+0.034 \mathrm{i}$ & 1.73 & 0.21 & 11 & $2.120+0.024 \mathrm{i}$ & 2.46 & 2.03 \\
12 & $2.110+0.042 \mathrm{i}$ & 2.68 & 1.42 & 12 & $2.158+0.013 \mathrm{i}$ & 2.76 & 1.44 \\
13 & $2.166+0.024 \mathrm{i}$ & 2.92 & 2.22 & 13 & $2.145+0.023 \mathrm{i}$ & 2.88 & 1.52 \\
14 & $2.116+0.024 \mathrm{i}$ & 3.08 & 0.32 & 14 & $2.169+0.027 \mathrm{i}$ & 3.20 & 0.48 \\
15 & $2.148+0.025 \mathrm{i}$ & 3.15 & 0.63 & 15 & $2.152+0.026 \mathrm{i}$ & 3.25 & 1.03 \\
16 & $2.165+0.022 \mathrm{i}$ & 3.22 & 2.40 & 16 & $2.116+0.031 \mathrm{i}$ & 3.84 & 2.66 \\
17 & $2.127+0.030 \mathrm{i}$ & 3.68 & 0.60 & 17 & $2.1769+0.001 \mathrm{i}$ & 0.901 & 0.866 \\
18 & $2.162+0.031 \mathrm{i}$ & 3.80 & 1.67 & 18 & $2.1757+0.001 \mathrm{i}$ & 0.929 & 0.872 \\
\hline
\end{tabular}



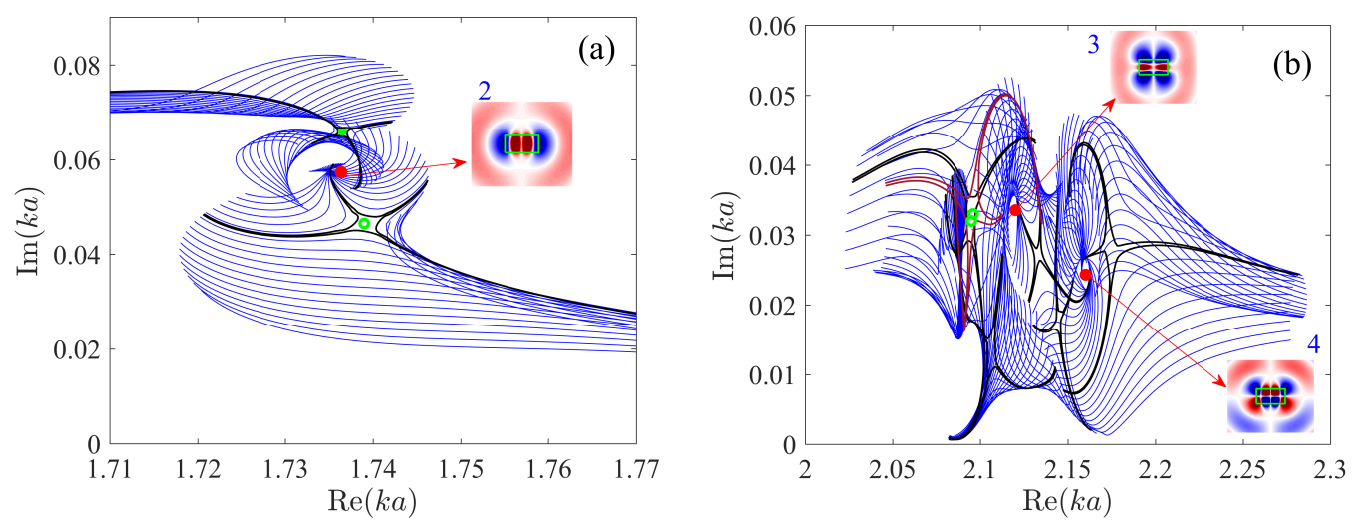

Figure 4. $(\mathbf{a}, \mathbf{b})$. Evolution of the eigenfrequencies of four disks for variation of the distances $1 \leq D \leq 2.5$ and $0.06 \leq L \leq 1.06$. Each line evolves with $L$ at fixed $D$. Red closed circles marks the eigenfrequencies of single disk with $h=1.04$ and $\epsilon=12$. Green open and closed circles mark symmetrical and anti-symmetrical EPs.
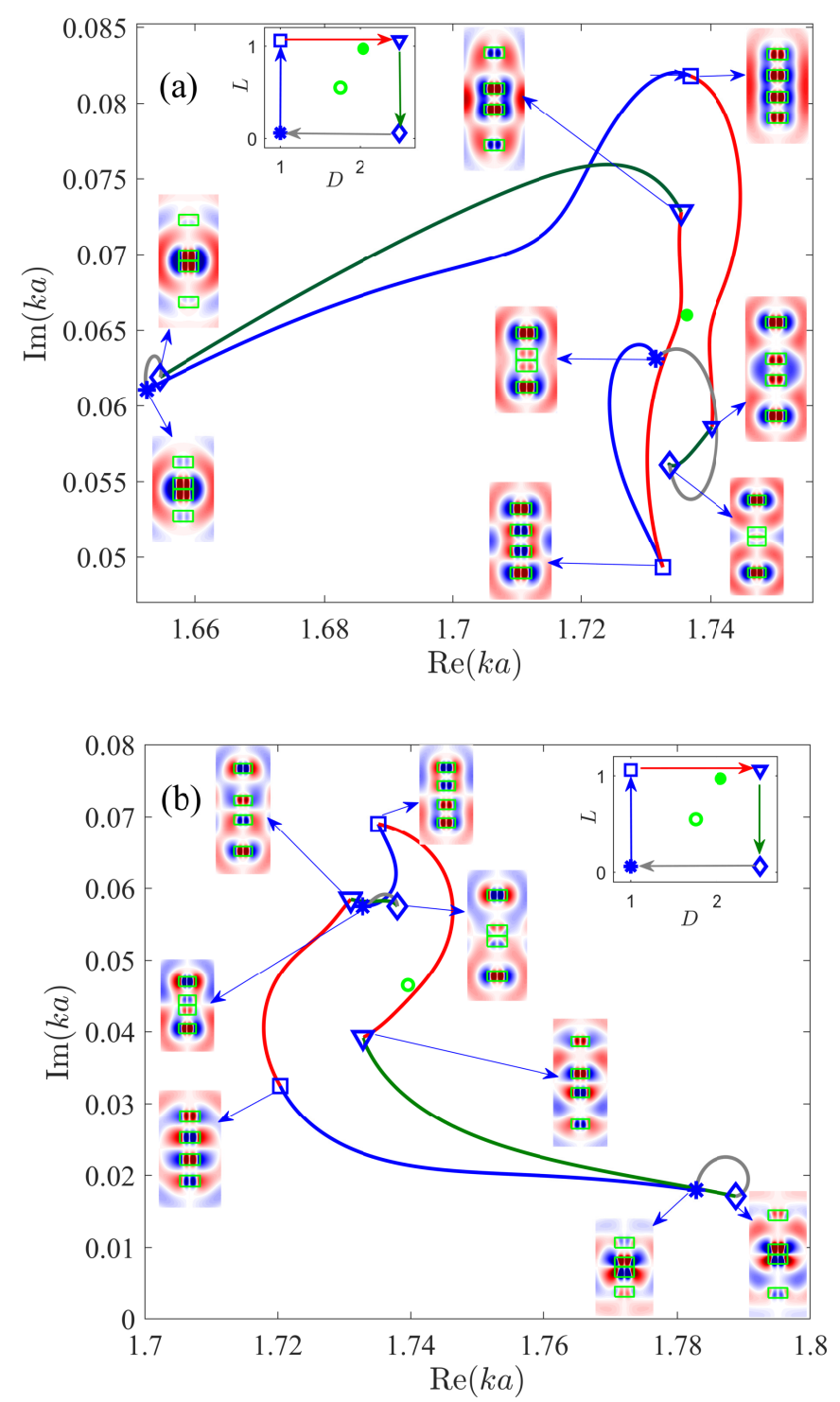

Figure 5. Encircling of the symmetric EP (a) and anti-symmetric EP (b) listed in Table 1 by the number 1 and marked by closed and open circles. The insets show the component $E_{\phi}$ of eigenmodes. 
Next, let us consider the family of EPs that originated from primary resonances 3 and 4 shown in the insets of Figure $3 c$ for variation of distances $D$ and L. Although in isolated disks the resonant modes 3 and 4 have opposite symmetry, in the total system of four disks, these resonances are coupled [44], which gives rise to a vast number of EPs of both symmetries listed in Table 1. EPs of each symmetry are to be considered separately because of their independence. The general theory of encircling of multiple EPs was developed in $[20,33,34,45-47]$ and was first observed in the system of four PT symmetrical coupled acoustic cylindrical resonators [34].

Although the number of EPs for encircling can be rather large, as follows from Table 1, we considered only the case of encircling of three symmetrical EPs enumerated as 5, 6, and 8 according to Table 1. Even for this case, we had seven scenarios to encircle three EPs. Figure 6 shows four principally different scenarios of encircling. We started with the encircling of EP 8 by a rectangular contour in the parametric space shown in the inset in Figure $6 a$ by green solid lines. The contour maps into the trajectory of the complex eigenfrequency shown by green lines too. The first closed encircling in the space $L, D$ maps into the $k a$ trajectory shown by solid lines, which were not closed. Markers of each turn in the rectangular contour were mapped into the same markers in the $k a$ plane. Only after the second rectangular encircling in the parametric space was the eigenfrequency trajectory $k a$ closing, which is shown by the dash-dot green line in Figure 6a. The reader can follow the evolution of the corresponding eigenmodes in Table 2 where the colors of $E_{\phi}$ correspond to the colors of markers in Figure 6. Thus, that case brings nothing new compared to the former case of interacting resonant modes that originated from the disk's elementary resonance 2, shown in Figure 5. Figure $6 \mathrm{~b}$ shows the case of encircling of two EPs: 5 and 8. One can see that the encircling gave rise to two independent eigenfrequency trajectories shown by green solid and dash-dotted lines. The trajectories were closing after two encirclings in the parametric space with corresponding eigenmodes listed in Table 2. However, to encircle another pair of EPs: 6 and 8, we obtained different behavior of the $k a$ trajectories, as Figure 6c shows. They were closed only after three encirclings in the parametric space, which corresponds to an interchange of three eigenmodes [20,33]. At last, Figure $6 \mathrm{~d}$ presents the encircling of all three EPs that required four windings in the parametric space.
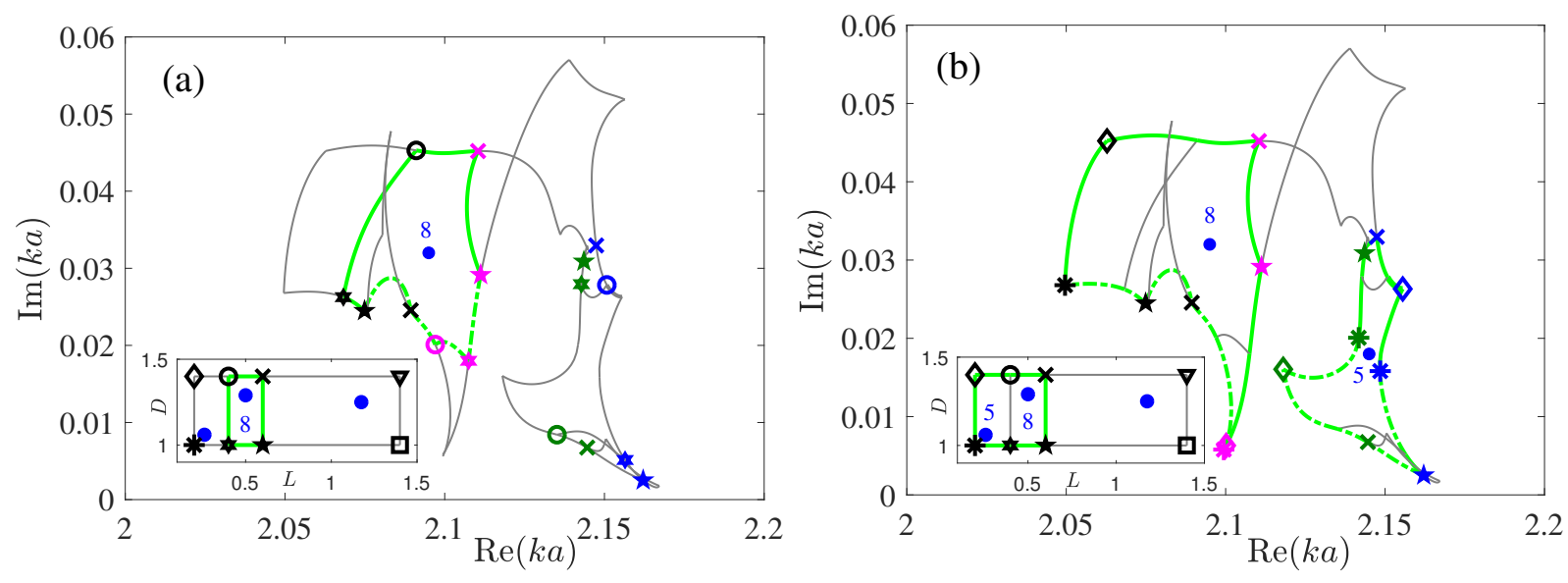

Figure 6. Cont. 

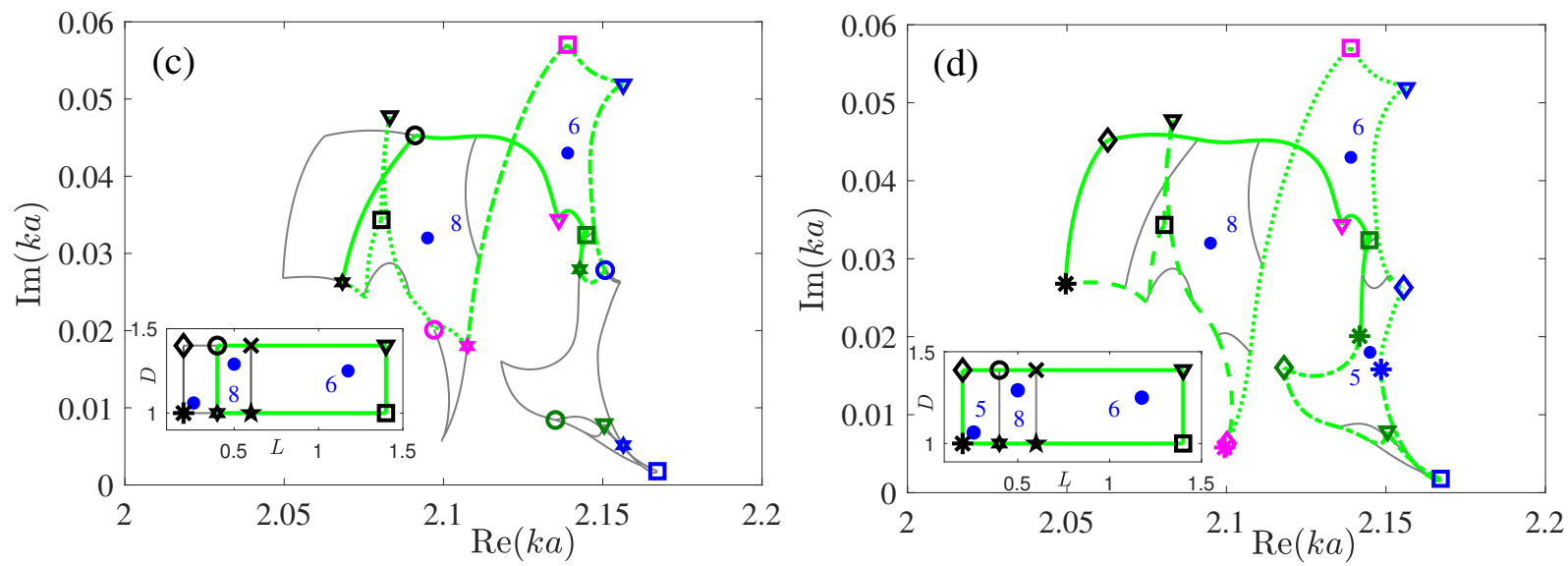

Figure 6. (a-d). Evolution of the eigenfrequencies of four disks for encircling in the two-dimensional parametric space $L, D$ shown in the insets. Each turn in the rectangular contour with corresponding markers was mapped into the same markers in the $k a$ space.

Table 2. $E_{\phi}$ component of EM field for symmetric eigenmodes of 4 disks.

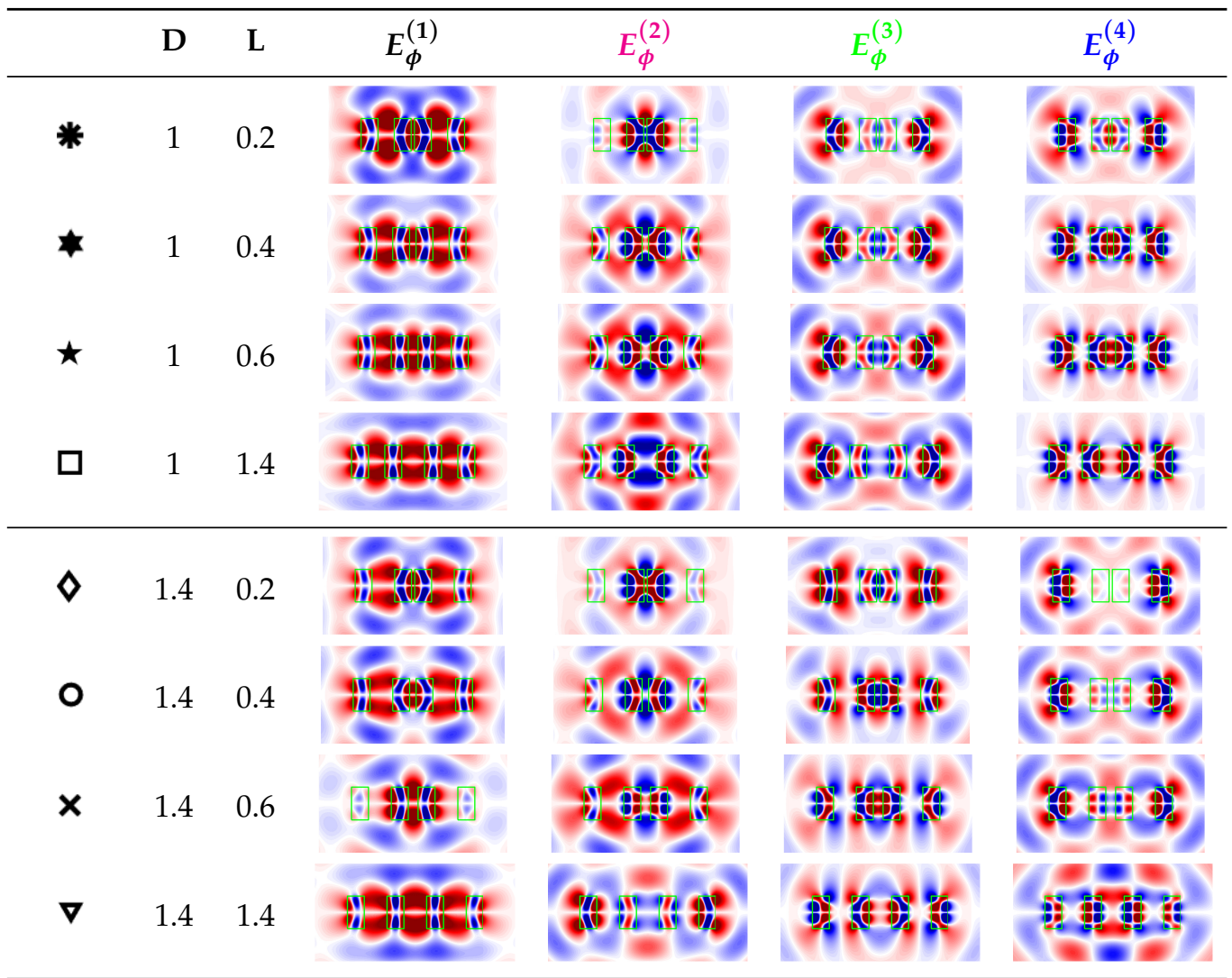

\section{Summary and Conclusions}

We demonstrated EPs in an isolated dielectric disk by variation of the cylindrical aspect ratio $h / a$ and its permittivity. However, in practice, that kind of variation challenges technological problems. In the present study, we considered the parametric space of two distances $L$ and $D$ between four identical silicon disks arranged in two dimers, as sketched in Figure 2. The isolated disk was given by elementary resonant modes shown in the insets of Figure 3. Each pair of disks spaced at a distance gave rise to the coupling of resonances of the disk in the form (1). All couplings between four disks resulted in the beautiful multi-spiral behavior of resonances, as plotted in Figure 3 for each elementary resonance. 
Then, the variation over two distances between dimers and disks in each dimer resulted in a few EPs due to avoided crossing of resonances originated from the elementary resonance 2 of the isolated disk (see Figure 3b).

Moreover, there were elementary resonances, enumerated 3 and 4 in Figure 3c, that were close each other. Although they had opposite symmetries relative to $z \rightarrow-z$, the presence of other disks removed the symmetry prohibition [44]. As a result, the effective non-Hermitian Hamiltonian can be presented by a matrix $8 \times 8$ similar to the matrix (2) with half of the eigenmodes symmetrical and half anti-symmetrical relative to inversion of the $z$-axis. For each kind of resonance complex, the eigenvalues showed substantially more complicated behavior with a vast number of EPs listed in Table 1. In the range of the distance $0.01<D<4$ (thickness of dimer) and the distance $0.01<L<4$ between dimers, we revealed 17 symmetrical EPs and 16 anti-symmetrical EPs.

The theory of encircling of multiple EPs was developed recently in refs. [20,33,34,45,47,48] and was applied in the case of four coupled acoustic resonators [34] and four microrings [48] with PT symmetry due to asymmetric gains and losses. In the present study, we showed a multiple number of symmetrical or anti-symmetrical EPs, which can be encircled by variation of two distances in the system of four coaxial silicon disks without PT symmetry. Being dependent on a method to encircle multiple EPs the initial eigenmodes recovered after two, three, and even four windings around these EPs, as shown in Figure 6. Note, we considered adiabatic encircling for which the state after encircling did not depend on the starting point. In dynamical encircling, however, the output state was predicted to be determined solely by the direction of rotation in the parameter space regardless of the input state, at least, for PT-symmetry systems [46,49].

Author Contributions: Conceptualization, A.S.; formal analysis, A.S.; investigation, K.P., E.B.; visualization, K.P.; writing—original draft preparation, K.P., A.S., E.B.; writing—review and editing, K.P., A.S., E.B. All authors have read and agreed to the published version of the manuscript.

Funding: This research was funded by Russian Foundation for Basic Research projects No. 19-0200055 .

Conflicts of Interest: The authors declare no conflict of interest.

\section{References}

1. Heiss, W.D.; Sannino, A.L. Avoided level crossing and exceptional points. J. Phys. A Math. Gen. 1990, 23, 1167-1178. [CrossRef]

2. Heiss, W. Phases of wave functions and level repulsion. Eur. Phys. J. D Atom. Mol. Opt. Phys. 1999, 7, 1-4. [CrossRef]

3. Feng, L.; Zhu, X.; Yang, S.; Zhu, H.; Zhang, P.; Yin, X.; Wang, Y.; Zhang, X. Demonstration of a large-scale optical exceptional point structure. Opt. Express 2013, 22, 1760-1767. [CrossRef]

4. Brandstetter, M.; Liertzer, M.; Deutsch, C.; Klang, P.; Schöberl, J.; Türeci, H.E.; Strasser, G.; Unterrainer, K.; Rotter, S. Reversing the pump dependence of a laser at an exceptional point. Nat. Commun. 2014, 5, 4034. [CrossRef]

5. Longhi, S. Parity-time symmetry meets photonics: A new twist in non-Hermitian optics. EPL (Europhys. Lett.) 2017, $120,64001$. [CrossRef]

6. Feng, L.; El-Ganainy, R.; Ge, L. Non-Hermitian photonics based on parity-time symmetry. Nat. Photonics 2017, 11, 752-762. [CrossRef]

7. Oezdemir, Ş.K.; Rotter, S.; Nori, F.; Yang, L. Parity-time symmetry and exceptional points in photonics. Nat. Mater. 2019, 18, 783-798. [CrossRef]

8. Miri, M.A.; Alù, A. Exceptional points in optics and photonics. Science 2019, 363, 7709. [CrossRef] [PubMed]

9. Feng, L.; Wong, Z.J.; Ma, R.M.; Wang, Y.; Zhang, X. Single-mode laser by parity-time symmetry breaking. Science 2014, 346, 972-975. [CrossRef]

10. Ghosh, S.N.; Chong, Y.D. Exceptional points and asymmetric mode conversion in quasi-guided dual-mode optical waveguides. Sci. Rep. 2016, 6, 19837. [CrossRef] [PubMed]

11. Feng, L.; Ayache, M.; Huang, J.; Xu, Y.L.; Lu, M.H.; Chen, Y.F.; Fainman, Y.; Scherer, A. Nonreciprocal Light Propagation in a Silicon Photonic Circuit. Science 2011, 333, 729-733. [CrossRef] [PubMed]

12. Laha, A.; Dey, S.; Gandhi, H.K.; Biswas, A.; Ghosh, S. Exceptional Point and toward Mode-Selective Optical Isolation. ACS Photonics 2020, 7, 967-974. [CrossRef]

13. Chen, W.; Özdemir, Ş.K.; Zhao, G.; Wiersig, J.; Yang, L. Exceptional points enhance sensing in an optical microcavity. Nature 2017, 548, 192-196. [CrossRef] 
14. Zhen, B.; Hsu, C.W.; Igarashi, Y.; Lu, L.; Kaminer, I.; Pick, A.; Chua, S.L.; Joannopoulos, J.; Soljačić, M. Spawning rings of exceptional points out of Dirac cones. Nature 2015, 525, 354-358. [CrossRef]

15. Kamiński, P.M.; Taghizadeh, A.; Breinbjerg, O.; Mørk, J.; Arslanagić, S. Control of exceptional points in photonic crystal slabs. Opt. Lett. 2017, 42, 2866. [CrossRef]

16. Abdrabou, A.; Lu, Y.Y. Exceptional points of resonant states on a periodic slab. Phys. Rev. A 2018, 97, 063822. [CrossRef]

17. Abdrabou, A.; Lu, Y.Y. Exceptional points of Bloch eigenmodes on a dielectric slab with a periodic array of cylinders. Phys. Scr. 2020, 95, 095507. [CrossRef]

18. Min, S.Y.; Kim, J.Y.; Yu, S.; Menabde, S.G.; Jang, M.S. Exceptional Points in Plasmonic Waveguides Do Not Require Gain or Loss. Phys. Rev. Appl. 2020, 14, 054041. [CrossRef]

19. Popov, V.; Tretyakov, S.; Novitsky, A. Brewster effect when approaching exceptional points of degeneracy: Epsilon-near-zero behavior. Phys. Rev. B 2019, 99, 045146. [CrossRef]

20. Ryu, J.W.; Lee, S.Y.; Kim, S.W. Analysis of multiple exceptional points related to three interacting eigenmodes in a non-Hermitian Hamiltonian. Phys. Rev. A 2012, 85, 042101. [CrossRef]

21. Kullig, J.; Yi, C.H.; Hentschel, M.; Wiersig, J. Exceptional points of third-order in a layered optical microdisk cavity. New J. Phys. 2018, 20, 083016. [CrossRef]

22. Yi, C.H.; Kullig, J.; Hentschel, M.; Wiersig, J. Non-Hermitian degeneracies of internal-external mode pairs in dielectric microdisks. Photonics Res. 2019, 7, 464. [CrossRef]

23. Huang, Y.; Shen, Y.; Veronis, G. Non-PT-symmetric two-layer cylindrical waveguide for exceptional-point-enhanced optical devices. Opt. Express 2019, 27, 37494. [CrossRef]

24. Abdrabou, A.; Lu, Y.Y. Exceptional points for resonant states on parallel circular dielectric cylinders. J. Opt. Soc. Am. B 2019, 36, 1659. [CrossRef]

25. Unterhinninghofen, J.; Wiersig, J.; Hentschel, M. Goos-Hänchen shift and localization of optical modes in deformed microcavities. Phys. Rev. E 2008, 78, 016201. [CrossRef] [PubMed]

26. Kullig, J.; Wiersig, J. Perturbation theory for asymmetric deformed microdisk cavities. Phys. Rev. A 2016, 94, 043850. [CrossRef]

27. Kullig, J.; Yi, C.H.; Wiersig, J. Exceptional points by coupling of modes with different angular momenta in deformed microdisks: A perturbative analysis. Phys. Rev. A 2018, 98, 023851. [CrossRef]

28. Jiang, T.; Xiang, Y. Perturbation model for optical modes in deformed disks. Phys. Rev. A 2019, 99, 023847. [CrossRef]

29. Lee, S.B.; Yang, J.; Moon, S.; Lee, S.Y.; Shim, J.B.; Kim, S.W.; Lee, J.H.; An, K. Observation of an Exceptional Point in a Chaotic Optical Microcavity. Phys. Rev. Lett. 2009, 103, 134101. [CrossRef] [PubMed]

30. Jiang, T.; Xiang, Y. Perfectly-matched-layer method for optical modes in dielectric cavities. Phys. Rev. A 2020, 102, 053704. [CrossRef]

31. Bulgakov, E.; Pichugin, K.; Sadreev, A. Exceptional points in dielectric spheroid. arXiv 2021, arXiv:2107.13719.

32. Kodigala, A.; Lepetit, T.; Kanté, B. Exceptional points in three-dimensional plasmonic nanostructures. Phys. Rev. B 2016, 94, 201103. [CrossRef]

33. Lee, S.Y.; Ryu, J.W.; Kim, S.W.; Chung, Y. Geometric phase around multiple exceptional points. Phys. Rev. A 2012, 85, 064103. [CrossRef]

34. Ding, K.; Ma, G.; Xiao, M.; Zhang, Z.; Chan, C. Emergence, Coalescence, and Topological Properties of Multiple Exceptional Points and Their Experimental Realization. Phys. Rev. X 2016, 6, 021007. [CrossRef]

35. Pick, A.; Zhen, B.; Miller, O.; Hsu, C.W.; Hernandez, F.; Rodriguez, A.; Soljačić, M.; Johnson, S. General theory of spontaneous emission near exceptional points. Opt. Express 2017, 25, 12325. [CrossRef] [PubMed]

36. Rybin, M.V.; Koshelev, K.L.; Sadrieva, Z.F.; Samusev, K.B.; Bogdanov, A.A.; Limonov, M.F.; Kivshar, Y.S. High-Q Supercavity Modes in Subwavelength Dielectric Resonators. Phys. Rev. Lett. 2017, 119, 243901. [CrossRef]

37. Pichugin, K.N.; Sadreev, A.F. Interaction between coaxial dielectric disks enhances the Q factor. J. Appl. Phys. 2019, 126, 093105. [CrossRef]

38. Bogdanov, A.A.; Koshelev, K.L.; Kapitanova, P.V.; Rybin, M.V.; Gladyshev, S.A.; Sadrieva, Z.F.; Samusev, K.B.; Kivshar, Y.S.; Limonov, M.F. Bound states in the continuum and Fano resonances in the strong mode coupling regime. Adv. Photonics $2019,1,1$. [CrossRef]

39. Odit, M.; Koshelev, K.; Gladyshev, S.; Ladutenko, K.; Kivshar, Y.; Bogdanov, A. Observation of Supercavity Modes in Subwavelength Dielectric Resonators. Adv. Mater. 2020, 33, 2003804. [CrossRef]

40. Awai, I.; Zhang, Y. Coupling coefficient of resonators-An intuitive way of its understanding. Electron. Commun. Jpn. (Part II Electron.) 2007, 90, 11-18. [CrossRef]

41. Elnaggar, S.Y.; Tervo, R.J.; Mattar, S.M. General expressions and physical origin of the coupling coefficient of arbitrary tuned coupled electromagnetic resonators. J. Appl. Phys. 2015, 118, 194901. [CrossRef]

42. Dembowski, C.; Graf, H.D.; Harney, H.L.; Heine, A.; Heiss, W.D.; Rehfeld, H.; Richter, A. Experimental Observation of the Topological Structure of Exceptional Points. Phys. Rev. Lett. 2001, 86, 787-790. [CrossRef] [PubMed]

43. Stehmann, T.; Heiss, W.D.; Scholtz, F.G. Observation of exceptional points in electronic circuits. J. Phys. A Math. Gen. 2004, 37, 7813-7819. [CrossRef]

44. Bulgakov, E.; Pichugin, K.; Sadreev, A. Mie Resonance Engineering in Two Disks. Photonics 2021, 8, 49. [CrossRef]

45. Demange, G.; Graefe, E.M. Signatures of three coalescing eigenfunctions. J. Phys. A Math. Theor. 2011, 45, 025303. [CrossRef] 
46. Zhang, X.L.; Wang, S.; Hou, B.; Chan, C. Dynamically Encircling Exceptional Points: In situ Control of Encircling Loops and the Role of the Starting Point. Phys. Rev. X 2018, 8, 021066. [CrossRef]

47. Jaiswal, R.; Banerjee, A.; Narayan, A. Characterizing and Tuning Exceptional Points Using Newton Polygons. arXiv 2021, arXiv:2107.11649.

48. Zhong, Q.; Khajavikhan, M.; Christodoulides, D.N.; El-Ganainy, R. Winding around non-Hermitian singularities. Nat. Commun. 2018, 9, 4808. [CrossRef]

49. Uzdin, R.; Mailybaev, A.; Moiseyev, N. On the observability and asymmetry of adiabatic state flips generated by exceptional points. J. Phys. A Math. Theor. 2011, 44, 435302. [CrossRef] 